\title{
Impact of Mobility on the Performance of Relaying in Ad Hoc Networks - Extended Version
}

\author{
A. Al Hanbali ${ }^{a, b}$, A. A. Kherani ${ }^{c}$, R. Groenevelt ${ }^{d}$, P. Nain ${ }^{b}$, and E. Altman ${ }^{b}$ \\ $a$ Université de Nice-Sophia Antipolis, department of Computer Science, Nice, France. \\ $b$ INRIA, 2004 Route des Lucioles, B.P.-93, Sophia-Antipolis Cedex, France, 06902. \\ $c$ Dept. of Computer Science and Engineering, IIT Delhi, New Delhi, India. \\ $d$ Accenture Technology Park, 449, Route des Cretes, Sophia Antipolis Cedex, France, 06902.
}

\begin{abstract}
We consider a mobile ad hoc network consisting of three types of nodes: source, destination, and relay nodes. All the nodes are moving over a bounded region with possibly different mobility patterns. We introduce and study the notion of relay throughput, i.e. the maximum rate at which a node can relay data from the source to the destination. Our findings include the results that a) the relay throughput depends on the node mobility pattern only via its (stationary) node position distribution, and b) that a node mobility pattern that results in a uniform steady-state distribution for all nodes achieves the lowest relay throughput. Random Waypoint and Random Direction mobility models in both one and in two dimensions are studied and approximate simple expressions for the relay throughput are provided. Finally, the behavior of the relay buffer occupancy is examined for the Random Walk and Random Direction mobility models. For both models, the explicit form of the mean buffer are provided in the heavy-traffic case.
\end{abstract}

\section{INTRODUCTION}

In mobile ad hoc networks (MANETs), since there is no fixed infrastructure and nodes are mobile, routes between nodes are set up and turn down dynamically. For this reason, MANETs often experience route failures and network disconnectivity which induce two of the main problems of MANETs. In order to overcome these problems, Grossglauser and Tse [9] proposed to exploit the node mobility in MANETs to increase the network throughput. Their idea was to look at the diversity gain achieved by using the mobile nodes as relays.

The relay mechanism proposed in [9], called the two-hop relay mechanism, is simple: if there is no route between the source node $(s)$ and the destination node $(d)$, the source node transmits its packets to one of its neighboring nodes (say, $r$ ) for delivery to the node $d$. This mechanism belongs to the family of routing protocols introduced in disruption tolerant networks (DTNs) [1].

In [5] it was then shown that a bounded delay can be guaranteed under the two-hop relay mechanism. The aim of these studies (see also [10]) is the scaling property of the throughput or delay as the number of nodes in the network becomes large. Our interest in the present work is in the performance of the above mentioned relay mechanism in a network consisting of a fixed and finite number of nodes. It was then shown in [5] that a bounded delay can be guaranteed under this relaying mechanism. The aim of these studies (see

An initial version of this work appeared in [2] also [10]) is the scaling property of the throughput or delay as the number of nodes in the network becomes large. Our interest in the present work is in the performance of the above mentioned relay mechanism in a network consisting of a fixed and finite number of nodes.

It is important to mention that most of the studies of scaling laws of delay or throughput in wireless ad hoc networks assume a uniform spatial distribution of nodes, which is the case, for example, when the nodes perform a symmetric Random Walk over the region of interest [5], [9]. In the present paper, we study the effect of the node mobility pattern on the throughput and delay performance of the relaying scheme of [9]. We are interested in the maximum relay throughput of a mobile node, i.e., the maximum that a node can contribute as a relay to the communication between two other nodes. The relaying of data for other nodes requires a relay node to allocate its own resources. In particular, a relay node has to keep the data to be relayed in its buffer. Hence, the study of the buffer behavior of a relay node forms an important topic of research. The present work addresses the above two issues, i.e., the maximum relay throughput and the relay node buffer behavior.

Our point of departure is a simple observation which relates the evolution of a relay node buffer at certain time instants, called cycle times, to the evolution of the workload process in a G/G/1 queueing system. The service requirements and inter-arrival times in this queueing system are determined by the characteristics of the mobility pattern of the nodes.

Our main findings are the following:

1) The relay throughput depends only on the stationary distribution of the nodes' position. Hence, any two mobility patterns that have the same stationary distribution will achieve the same relay throughput.

2) It is assumed in [9] that the stationary distribution of a node position is uniform over the region of interest. This has led to many research efforts which base their work on this particular assumption [5], [10]. We prove that the relay throughput achieved is the lowest when nodes are uniformly distributed.

3) Knowledge of the stationary node location distribution alone is not enough to understand the behavior of relay node buffer at cycle times. A detailed analysis involving second-order moments of contact times between mobile nodes is necessary to obtain a full picture. We perform 
such an analysis for the Random Direction model [15] inside a square in addition to the random walk model over a circle derived in [2].

4) We then show numerically that the average relay buffer size at cycle times derived in [2, Sec. VI] converges to the time average of relay buffer size as the workload at the relay node increases. This result was validated for the Random Direction and Random Waypoint mobility models inside a square [4].

An important point that needs to be emphasized is that, unlike [5], [9], [10] which study the system performance when the number of nodes is large, we are interested in a relay node performance while it is involved in relaying data between two particular nodes. Developing models for performance analysis of a relay node buffer and the relay throughput can help in dimensioning a relay node buffer size and on achieving an optimal performance using relaying mechanisms. We note that the model studied in this paper is not restricted to three nodes, nor that the model requires the same mobility pattern for all of the nodes.

The rest of the paper is organized as follows: Section II describes the relaying system considered. In Section III we develop a queueing model for the relay buffer (RB). Section IV studies the effect of mobility models on the relaying throughput, and in Section $\mathrm{V}$ we find expressions for the relay throughput for the Random Waypoint and the Random Direction models in both one and in two dimensions. Section VI studies the RB behavior for the Random Walk and Random Direction models. In Section VII, we report numerical results on the stability, relay throughput, contact time distribution, probability of a 2-hop route, and the RB behavior. Section VIII concludes the paper and gives research directions.

\section{The System ModeL}

To study the maximum rate at which a node can relay data, we start by considering the scenario where three nodes move in a two-dimensional bounded region. One of these nodes is the source of packets, one is the destination, and the third one is the relaying node. The mobility patterns of the three nodes are independent and may be different from each other; this is in contrast with [5], [9] where the authors assume that the mobility pattern of the nodes is such that the steady-state distribution of the location of all the nodes is uniform over the region of interest. In fact, [5] assumes that nodes perform random walks (there are other mobility models which also result in a uniform stationary distribution, e.g., the Random Direction model). As mentioned earlier, we are interested in the maximum relay throughput of a relay node. As a starting point we will restrict ourselves to the case where there is only one relay node. At a later stage we will relax this assumption. Also, we want to study the dependence of the relay node buffer behavior on the mobility model. We assume that a node detects its one-hop neighbor(s) by sending periodically Hello messages. However to detect two-hop neighbors, nodes exchange the addresses of their neighbors.

The model is the following:
1) The three nodes move independently of each other according to a (possibly node-dependent) mobility model inside a bounded 2-dimensional region.

2) The source node has always data to send to the destination node. This is a standard assumption, also made in [5], [9], [10], because we are interested in the maximum relay throughput of the relay node.

3) When the relay node comes within the transmission range of the source node (we will also say that nodes are in contact in this case), and if the destination node is outside the transmission range of the source and of the relay node, then the relay node accrues packets to be relayed to the destination node at a constant rate $r_{s}$. [We could allow for a stochastic nature of traffic generated by the source by assuming that $r_{s}$ is an independent stochastic process. However, such a study is out of the scope of this work.]

4) When the destination node comes within the transmission range of the relay node, and if the destination and the relay node are outside transmission range of the source node, then the relay node sends the relay packets (if any) to the destination node at a constant rate $r_{d}$.

5) If the relay node is within transmission range of both the source node and the destination node, then the relay node does not contribute to relaying. In this case there is either a direct communication between the source and destination or there is a two-hop route via the relay node so that the relay node acts as a forwarding node and not as a relay.

Our objective is to study the properties of the relay buffer (stability, stationary occupancy distribution, throughput). To this end, we first develop a queueing model that will give many insights into the system behavior.

\section{A Queueing Model For THE Relay Buffer}

After addressing the case where there are only three mobile nodes in Section III-A, we investigate the situation of an arbitrary number of source/destination/relay nodes, under the additional assumption that all source and destination nodes are fixed (cf. Section III-B).

\section{A. Single Source, Destination, and Relay Nodes}

The state of the relay node at time $t$ is represented by the random variable (r.v.) $S_{t} \in\{-1,0,1\}$ where:

- $S_{t}=1$ if at time $t$ the relay node is neighbor (i.e., within transmission range) of the source, and if the destination is a neighbor neither of the source nor of the relay node. In other words, when $S_{t}=1$, the source node sends relay packets to the relay node at time $t$;

- $S_{t}=-1$ if at time $t$ the relay node is a neighbor of the destination, and if the source is a neighbor neither of the destination nor of the relay node. When $S_{t}=$ -1 the relay node delivers relay packets (if any) to the destination;

- $S_{t}=0$ otherwise. 
Mobiles have finite speeds. We will assume that the relay node may only enter state 1 (resp. -1 ) from state 0 : if $S_{t-} \neq S_{t}$ then necessarily $S_{t}=0$ if $S_{t-}=1$ or $S_{t-}=-1$.

Denote by $B_{t}$ the RB occupancy at time $t$. The r.v. $B_{t}$ evolves as follows:

- it increases at rate $r_{s}$ if $S_{t}=1$. This is because when $S_{t}=1$, the relay node receives data to be relayed from the source node at rate $r_{s}$;

- it decreases at rate $r_{d}$ if $S_{t}=-1$ and if the RB is nonempty. This is because if $S_{t}=-1$, and if there is any data to be relayed, then the relay node sends data to the destination node at rate $r_{d}$.

- it remains unchanged in all other cases.

Let $\left\{Z_{n}\right\}_{n}\left(Z_{1}<Z_{2}<\cdots\right)$ denote the consecutive jump times of the process $\left\{S_{t}, t \geq 0\right\}$. An instance of the evolution of $S_{t}$ and $B_{t}$ as a function of $t$ is displayed in Figure 1.

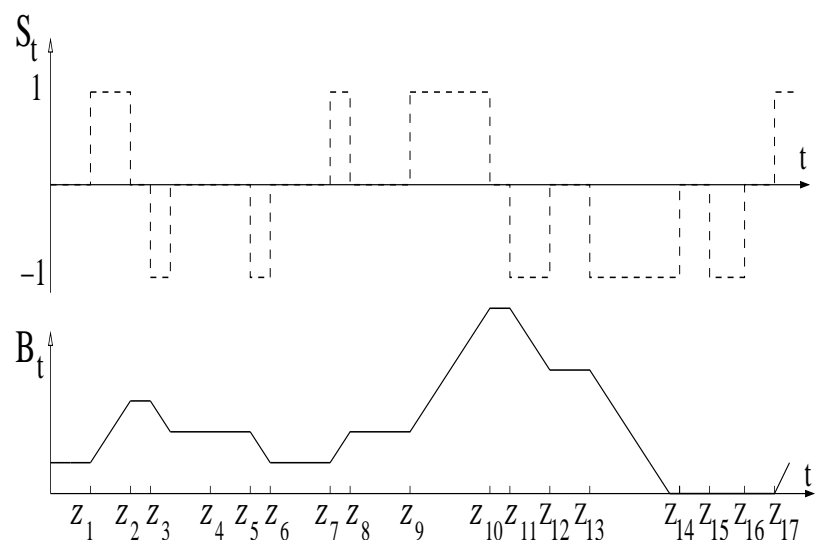

Fig. 1. Evolution of $\left\{S_{t}\right\}_{t}$ and relay node buffer occupancy.

The evolution of the discrete indexed process $\left\{S_{Z_{k}}, k \geq 1\right\}$ consists of sequences of 1,0 and -1 . This naturally motivates us to look at the times when the relay node returns to the source node after being neighbor of the destination node at least once. This is done in the following.

We define a cycle as the interval of time that starts at $t=$ $Z_{k}$, for some $k$ with $S_{t}=1$, and (necessarily) $S_{t-}=0$ and $S_{Z_{k-2}}=-1$, and ends at the smallest time $t+\tau$ such that $S_{t+\tau}=1$ and $S_{t+s}=-1$ for some $s<\tau$. In Figure 1, the time-interval $\left[Z_{7}, Z_{17}\right)$ constitutes a cycle. Let $C_{n}$ denotes the duration of the $\mathrm{n}^{\text {th }}$ cycle. Note that there is no restriction on the number of times the relay node becomes neighbor of the source node or of the destination node during a cycle. Hence, during a cycle the relay node will transmit packets to the destination and will receive packets from the source. Also, we note that the end time of the $\mathrm{n}^{\text {th }}$ cycle is also the start time of the $(\mathrm{n}+1)^{t h}$ cycle.

Let $W_{n}$ be the time at which the $n^{\text {th }}$ cycle begins. Let

$$
\sigma_{n} \triangleq \int_{t=W_{n}}^{W_{n+1}} \mathbf{1}_{\left\{S_{t}=1\right\}} d t
$$

be the amount of time spent by the relay node in state 1 during the $n^{\text {th }}$ cycle. Similarly, let

$$
\alpha_{n} \triangleq \int_{t=W_{n}}^{W_{n+1}} \mathbf{1}_{\left\{S_{t}=-1\right\}} d t
$$

be the amount of time spent by the relay node in state -1 during the $n^{t h}$ cycle. Observe that during the amount of time $\sigma_{n}$, the RB increases at rate $r_{s}$, and it decreases at rate $r_{d}$ during the amount of time $\alpha_{n}$. Let $\tilde{B}_{n}$ be the RB occupancy at the beginning of the $n^{\text {th }}$ cycle, i.e. $\tilde{B}_{n}=B_{W n}$. Clearly,

$$
\tilde{B}_{n+1}=\left[\tilde{B}_{n}+r_{s} \sigma_{n}-r_{d} \alpha_{n}\right]^{+}
$$

where $[x]^{+}=\max (x, 0)$. In other words, $\tilde{B}_{n+1}$ can be interpreted as the workload seen by the $(n+1)^{s t}$ arrival in a G/G/1 queue, where $r_{s} \sigma_{n}$ is the service requirement of the $n^{\text {th }}$ customer, and $r_{d} \alpha_{n}$ is the inter-arrival time between the $n^{\text {th }}$ and the $(n+1)^{\text {st }}$ customer. This interpretation will be used next.

Assumption A: Throughout Section III-A we assume that the sequence $\left\{C_{n}, \sigma_{n}, \alpha_{n}\right\}_{n}$ is stationary and ergodic, with $0<E\left[C_{n}\right]<\infty, 0<E\left[\sigma_{n}\right]<\infty$ and $0<E\left[\alpha_{n}\right]<\infty$.

Clearly, the statistical properties of the random variables $C_{n}$, $\sigma_{n}$, and $\alpha_{n}$ will depend on the node mobility patterns. Hence, our study will be restricted to the class of mobility models under which the stationarity and ergodicity assumptions hold for the sequence $\left\{C_{n}, \sigma_{n}, \alpha_{n}\right\}_{n}$.

Definition: The long-term fraction of time the $\mathrm{RB}$ receives data is

$$
\pi_{s} \triangleq \lim _{t \rightarrow \infty} \frac{1}{t} \int_{u=0}^{t} \mathbf{1}_{\left\{S_{u}=1\right\}} d u
$$

and the long-term fraction of time that the destination node is the neighbor of only the relay node ( i.e., the fraction of time that the RB may drain off) is

$$
\pi_{d} \triangleq \lim _{t \rightarrow \infty} \frac{1}{t} \int_{u=0}^{t} \mathbf{1}_{\left\{S_{u}=-1\right\}} d u .
$$

It can be shown that these limits exist under Assumption A. The proof is beyond the scope of this work. Moreover, Assumption A implies that [3]

$$
\pi_{s}=\lim _{t \rightarrow \infty} P\left(S_{t}=1\right)=\frac{E\left[\sigma_{n}\right]}{E\left[C_{n}\right]}
$$

and

$$
\pi_{d}=\lim _{t \rightarrow \infty} P\left(S_{t}=-1\right)=\frac{E\left[\alpha_{n}\right]}{E\left[C_{n}\right]} .
$$

Theorem 1: If $r_{s} E\left[\sigma_{n}\right]<r_{d} E\left[\alpha_{n}\right]$ then $\tilde{B}_{n}$ converges in probability to a proper and finite r.v. $\widetilde{B}$ (i.e., $\lim _{n} P\left(\tilde{B}_{n}<x\right)$ $=P(\tilde{B}<x) \forall x \geq 0)$. If $r_{s} E\left[\sigma_{n}\right]>r_{d} E\left[\alpha_{n}\right]$, then $\tilde{B}_{n}$ converges in probabilty to $+\infty$ almost surely.

Proof. Follows from the relation to the G/G/1 queue made above and [14].

Remark 1: In terms of $\pi_{s}$ and $\pi_{d}$, the stability condition of Theorem 1 writes

$$
r_{s} \pi_{s}<r_{d} \pi_{d}
$$

Remark 2: If all nodes have the same mobility pattern, then clearly $\pi_{s}=\pi_{d}$, since the relay node is equally likely to be within the transmission range of the source and of the destination. Therefore, by Remark 1, the stability condition is

$$
r_{s}<r_{d}
$$


Theorem 2: If $r_{s} \pi_{s}<r_{d} \pi_{d}$, then the relay throughput $T_{r}$, defined as the stationary output rate of the relay node, is given by

$$
T_{r}=r_{s} \pi_{s} .
$$

Proof. In steady-state the RB can be thought of as a standard $\mathrm{G} / \mathrm{G} / 1$ queue so that the output rate is the same as the input rate and is given by $r_{s} \pi_{s}$.

Remark 3: The relay throughput $T_{r}$ only depends on $r_{s}$ and the stationary distribution of the node mobility pattern. In particular, two different mobility patterns with the same stationary distribution (for the location of the nodes) will yield the same relay throughput.

It is clear from Theorem 2 and Remark 1 that $\pi_{s}$ and $\pi_{d}$ play an important role in determining the stability and the throughput of the RB. Much of the rest of this paper will be devoted to the study of these quantities.

\section{B. Multiple Source, Destination, and Relay Nodes}

We now assume that there are $K$ source nodes, $M$ destination nodes and $N$ relay nodes, all with the same transmission range $R$ (the latter will be assumed throughout). The source and destination nodes are stationary. The relay nodes move independently of each other inside a connected area $A$ according to a common mobility model. The distance between any two source nodes, and between any two destination nodes, is assumed to be greater than $2 R$. This implies that a relay node can not receive (resp. transmit) data from (resp. to) two or more source (resp. destination) nodes at the same time.

Furthermore, assume that the routing protocol generates routes of length no more than $h$-hops, i.e., the lifetime of a packet in number of hops is not greater than $h$. The distance between any source and any destination node is set to be greater than $h R$. Therefore, there does not exist a direct route from any source to any destination node, which implies that packets of a source have to use the mobile relay nodes in order to be transfered to their destinations.

The RB of a relay node is composed of $M$ queues; one for each of the $M$ destinations. The system behaves as follows:

1) When there are $i$ relay nodes inside the transmission range of source node $k$, where $i \in\{1, \cdots, N\}$ and $k \in\{1, \cdots, K\}$, then the source transmits to the $i$ relay nodes the packets addressed to destination node $m \in\{1, \cdots, M\}$ with probability $P_{k}^{m}$ in a round-robin scenario, where $\sum_{m=1}^{M} P_{k}^{m}=1$. Note that we assume that there is only one copy of the packet. Thus queue $m$ of the relay node accrues packets at a fixed rate $r_{S_{k}} P_{k}^{m} / i$, where $r_{S_{k}}$ is the transmission rate of source node $k$.

2) When the relay node receives a packet from a source that is destined to destination node $m$, it buffers this packet in its queue of index $m$.

3) When there are $j$ relay nodes with non-empty queue $m$ inside the transmission range of destination $m$, these relay nodes share the channel bandwidth fairly. More precisely, queue $m$ of these $j$ relay nodes drains off at a fixed rate $r_{D_{m}} / j$, where $r_{D_{m}}$ is the transmission rate of a relay node to the destination node $m$. The service discipline in queue $m$ of the relay node is FIFO.

Let $f(x), x \in A$, be the stationary node location probability density. Denote by $x_{S_{k}}$ and $x_{D_{m}}$ the fixed location in $A$ of source $k \in\{1, \cdots, K\}$ and destination $m \in\{1, \cdots, M\}$, respectively.

Hence, the probability that a relay node is the neighbor of a node located in $x \in A$ is

$$
\pi(x)=\int_{\{y \in A: d(x, y) \leq R\}} f(y) d y,
$$

where $d(u, v)$ is the Euclidean distance between locations $u$ and $v$.

By conditioning on the number of nodes within range of source node $k$, we find that the input rate at queue $m$ of each relay node is

$$
\begin{aligned}
\tau_{S_{k}}^{m} & =P_{k}^{m} r_{S_{k}} \sum_{i=1}^{N} \frac{1}{i}\left(\begin{array}{c}
N-1 \\
i-1
\end{array}\right) \pi\left(x_{S_{k}}\right)^{i}{\overline{\pi\left(x_{S_{k}}\right)}}^{N-i} \\
& =P_{k}^{m} r_{S_{k}} \frac{1-\left(1-\pi\left(x_{S_{k}}\right)\right)^{N}}{N},
\end{aligned}
$$

where $\bar{a}:=1-a$.

The overall long-term arrival rate to queue $m$ of a relay node from all of the sources is

$$
\tau_{S}^{m}:=\sum_{k=1}^{K} \tau_{S_{k}}^{m}=\frac{1}{N} \sum_{k=1}^{K} P_{k}^{m} r_{S_{k}}\left[1-\left(1-\pi\left(x_{S_{k}}\right)\right)^{N}\right]
$$

The exact derivation of $\tau_{D}^{m}$, the long-term service rate of queue $m$ at a relay node, is intractable since it depends on the (stationary distribution of the) location of the other relay nodes with respect to the destination $D_{m}$, and on whether or not queue $m$ at each relay node is empty or not and located within transmission range of $D_{m}$. More precisely, if $i$ relay nodes are within the transmission range of destination $D_{m}$, and if queue $m$ in each of these relay nodes is non-empty, then the service rate in queue $m$ at each of the $i$ relay nodes is $r_{D_{m}} / i$. The above reasoning indicates that $r_{D_{m}} / N$ is the minimum instantaneous service rate at each queue $m$. This yields the following lower bound-called $\hat{\tau}_{D}^{m}$-on the longterm service rate of queue $m$ :

$$
\hat{\tau}_{D}^{m}=r_{D_{m}} \frac{1-\left(1-\pi\left(x_{D_{m}}\right)\right)^{N}}{N} .
$$

As a result, a sufficient condition for the stability of queue $m$ at each relay node is

$$
\tau_{S}^{m}<\hat{\tau}_{D}^{m}
$$

If queue $m$ at a relay node is stable, then the relay throughput $T_{r}^{m}$ at this queue is equal to its long-term arrival rate, i.e,

$$
T_{r}^{m}=\tau_{S}^{m}=\sum_{k=1}^{K} \tau_{S k}^{m}=\frac{1}{N} \sum_{k=1}^{K} P_{k}^{m} r_{S k}\left[1-{\overline{\pi\left(x_{S k}\right)}}^{N}\right] .
$$


The network throughput, $T$, is the sum of the relay throughputs at all the $M$ queues of all the $N$ relay nodes, namely

$$
T=\sum_{n=1}^{N} \sum_{m=1}^{M} T_{r}^{m}=\sum_{k=1}^{K} r_{S k}\left[1-\left(1-\pi\left(x_{S k}\right)\right)^{N}\right] .
$$

Observe that $1-\left(1-\pi\left(x_{S k}\right)\right)^{N}$ is the probability that there is at least one relay node inside the transmission range of the source node $k$.

We conclude this section by briefly addressing the situation where all of the nodes are moving. Since an exact calculation of the throughput of queue $m$ at a relay node is very difficult, we will derive an approximation for this quantity. This approximation is based on the assumption that routes cannot exceed two hops. We assume that all nodes move independently of each other with the same mobility pattern, and that they have the same transmission range. Let $p_{1}$ be the probability that two nodes are within transmission range of one another. Let $p_{2}$ be the probability that three nodes constitute a two-hop route. Then, under the above simplifying assumption

$$
\begin{aligned}
& \sum_{i=1}^{N} \frac{P_{k}^{m} r_{S_{k}}}{i}\left(\begin{array}{c}
N-1 \\
i-1
\end{array}\right)\left(p_{1}-p_{2}\right)^{i}\left(1-p_{1}\right)^{N+1-i} \\
= & P_{k}^{m} r_{S_{k}} \frac{\left(1-p_{1}\right)\left(\left(1-p_{2}\right)^{N}-\left(1-p_{1}\right)^{N}\right)}{N}
\end{aligned}
$$

is the contribution of source node $k$ to the long-term arrival rate in queue $m$ at any relay node. Therefore, the overall long-term input rate at queue $m$ at any relay node can be approximated by summing up the r.h.s. of the above identity over all the values of $k$. This gives

$$
\tau_{S}^{m} \approx \frac{\left(1-p_{1}\right)\left(\left(1-p_{2}\right)^{N}-\left(1-p_{1}\right)^{N}\right)}{N} \sum_{k=1}^{K} P_{k}^{m} r_{S_{k}} .
$$

When $P_{k}^{m}=1 / M$ (that is, there is a uniform probability that source node $k$ sends to destination node $m$ ) and when the transmission rates of all sources are equal to $r_{S}$, then (16) becomes

$$
\tau_{S}^{m} \approx r_{S} \frac{\left(1-p_{1}\right)\left(\left(1-p_{2}\right)^{N}-\left(1-p_{1}\right)^{N}\right)}{M N} .
$$

In the next section, we will investigate the impact of the mobility pattern on the relay throughput. We will show that the throughput is minimized when in steady-state the nodes are uniformly distributed over the area.

\section{COMPARISON OF MOBILITy MODEls}

We consider the scenario where nodes move independently of each other according to same mobility pattern. Assume that the nodes location distribution is stationary. The nodes position can take values in a discrete set $X$ with cardinality $\# X=G$. Let $G(x), x \in X$ denote the set of all points in the transmission range of a node located at $x$. We assume that there is complete symmetry, so that $\# G(x)=\# G(y)$ for all $x, y \in X$ and that if $x \in G(y)$ then $y \in G(x)$. This can be assumed when there is no boundary effect, for example, as is the case of motion over a torus or over a circle (representing, respectively, motion over a plane or line with wrap around).
Let $P$ be the probability measure over $X$ that represents the stationary node location distribution. As the cardinality of $X$ is equal to $G, P$ can be represented as an $G$-dimensional (column) vector. The uniform stationary node location over $X$, called $U$, is a $G$-dimensional vector whose entries are all equal to $\frac{1}{G}$. Let $e_{x}, x \in X$, denote a probability measure over $X$ which gives all mass to position $x$, i.e., $e_{x}$ is an $G$-dimensional vector whose entries are all equal to 0 except for the $x^{\text {th }}$ components which is equal to 1 .

For any stationary node location distribution $P$ over $X$, let $g(P)$ denote the probability that two nodes are neighbor of each other. Let $H$ denote the neighborhood matrix, i.e., $H_{x, y}=1$ if $y \in G(x)$ and $H_{x, y}=0$ otherwise. Note $H$ is a symmetric matrix. In terms of $P_{x}$ (resp. $P_{y}$ ), the probability that a node is at location $x$ (resp. $y$ ) in the stationary regime $g(P)$ writes

$$
g(P)=\sum_{x \in X} P_{x} \sum_{y \in G(x)} P_{y}=\sum_{x \in X} P_{x} \sum_{y \in X} H_{x, y} P_{y}=P^{T} H P
$$

where $P^{T}$ is the transpose of $P$ and we use the fact that the locations of the nodes are independent.

Theorem 3: A uniform distribution of nodes over the region of interest achieves the minimum probability of contact between any two nodes.

Proof: Consider any $P$ of the form

$$
P=U+\delta e_{x}-\delta e_{y},
$$

for some $0<\delta<1$ and $x, y \in X, x \neq y$. Then

$$
\begin{aligned}
g(P)= & P^{T} H P \\
= & g(U)+\delta^{2}\left(e_{x}^{T} H e_{x}+e_{y}^{T} H e_{y}-e_{x}^{T} H e_{y}-e_{y}^{T} H e_{x}\right) \\
& +2 \delta\left(e_{x}-e_{y}\right)^{T} H U
\end{aligned}
$$

where we have used the fact that for all $x \in X e_{x}^{T} H e_{x}=1$ as $x \in G(x)$. Since $H$ is a symmetric matrix, we have $P^{T} H Q=$ $Q^{T} H P$ for all $P, Q$ probability measures on $X$. Also, it is easy to see that $e_{y}^{T} H e_{x}=1$ if $x \in G(y)$ and is 0 otherwise. Hence we get

$$
\begin{aligned}
g(P) & =g(U)+2 \delta^{2}\left(1-\mathbf{1}_{\{x \in G(y)\}}\right)+2 \delta\left(e_{x}-e_{y}\right)^{T} H U \\
& =g(U)+2 \delta^{2} \mathbf{1}_{\{x \notin G(y)\}}+\frac{2 \delta(\# G(x)-\# G(y))}{G},
\end{aligned}
$$

where in the last expression we have used the, easy to observe, fact that $e_{x}^{T} H U=\frac{\# G(x)}{G}$. Hence, since $\# G(x)=\# G(y)$ for all $x, y \in X$, it is seen that $g(P)-g(U)=2 \delta^{2} \mathbf{1}_{\{\mathbf{x} \notin \mathbf{G}(\mathbf{y})\}} \geq$ 0. Which implies that

$$
U \in \underset{P=U+\delta e_{x}-\delta e_{y}}{\operatorname{argmin}} g(P) .
$$

Now, any other probability distribution over the set $X$ is a point in $G$-dimensional canonical simplex. The uniform distribution is at the centroid of this simplex and any other distribution $P$, when viewed as an $G$ dimensional vector (a point in the simplex), can be written as

$$
P=U+\epsilon,
$$

where $U$ is the uniform distribution and $\epsilon$ is an $G$-dimensional vector whose entries are in the interval $\left[-\frac{1}{G}, \frac{G-1}{G}\right]$ and the 
entries sum to zero. Clearly, any such $\epsilon$ can be written as a (possibly non-unique) finite sum

$$
\epsilon=\sum_{x \in I(P)}\left(e_{x}-e_{y(x)}\right) \delta_{x}
$$

where $I(P) \subset X$ is some index set, $y(x) \in X$, and $\delta_{x}>$ $0, x \in I(P)$. This is because $e_{x}$ forms a basis for the $G$-dimensional space and because $P$ is a probability vector with $\sum_{x \in X} \epsilon_{x}=0$.

Recall that if the stationary node distribution is $P$, we can write $g(P)$ as $g(P)=P^{T} H P$, where $H$ is an $G \times G$ symmetric matrix indicating the neighborhood relation. We have already shown that when $P=U$, the uniform distribution, the directional derivative of $P^{T} H P$ is positive along any direction of the form $\left(e_{x}-e_{y}\right)$ where $e_{x}$ is $G$-dimensional vector with all except the $x^{\text {th }}$ entry equal to zero. We now use continuity of the derivative of $g(U)$ to conclude that its directional derivative along any direction is positive. Hence $U \in \underset{P}{\operatorname{argmin}} g(P)$.

The above result does not imply that the relay throughput achieves its minimum under the uniform stationary node distribution. This is because the relay throughput under distribution $P$, denoted $T_{r}(P)=r_{s} \pi_{s}(P)$ and with $\pi_{s}(P)=$ $\lim _{t \rightarrow \infty} P\left(S_{t}=1\right)$ under the probability measure $P$, is

$$
T_{r}(P)=r_{s}\left(g(P)-\sum_{x \in X} P_{x} \sum_{y \in G(x)} P_{y} \sum_{z \in G(x) \cup G(y)} P_{z}\right),
$$

and it can be easily seen that for any $x \in X, \pi_{s}\left(e_{x}\right)=0$. Since $\pi_{s}(\cdot)$ is a probability, this implies that $P=e_{x}$ achieves minimum of $\pi_{s}(\cdot)$. However, it is reasonable to assume that the uniform distribution is a local minimum for $\pi_{s}(\cdot)$ because the second term in expression for $\pi_{s}(\cdot)$ above is of smaller order as compared to the first term.

Observe that if the source node and the destination node are fixed, and if they are far apart (so that a two-hop communication between them via a relay node is not possible), then a uniform distribution of relay node achieves the minimum relay throughput.

In the next section, we will find expressions for the relay throughput in the case nodes move according to the Random Waypoint and the Random Direction models.

\section{ThroughPUT IN RANDOM WAYPOINT AND RANDOM DIRECTION MODELS}

In this section, we compute the relay throughput in the case where (i) the relay node moves along a finite interval according either to the Random Waypoint model or to the Random Direction model, the source and destination nodes being stationary (Section V-A.1), (ii) all nodes move independently of each other, with the same mobility model (Random Direction or Random Waypoint), either along a finite interval (Section VA.2) or inside a square (Section V-B).

We have shown in Theorem 2 that the relay throughput $T_{r}$ is given by $T_{r}=r_{s} \pi_{s}$, where $r_{s}$ is the transmission rate of the source to the relay node ( $r_{s}$ is a given parameter), and $\pi_{s}$ is the stationary probability that the source is sending packets to the relay node (see Section III). In the following, we will compute $\pi_{s}$ for each case mentioned above. This will be carried out under the assumption that all nodes have the same transmission range $R$.

\section{A. One Dimension}

For the Random Waypoint mobility model over the interval $[0, L]$, the stationary probability density function of a node location is [4]

$$
f(x)=\frac{6(L-x) x}{L^{3}}, \quad x \in[0, L] .
$$

The stationary probability density function under the Random Direction mobility model is uniform [15], i.e.,

$$
f(x)=\frac{1}{L}, \quad x \in[0, L] .
$$

1) Only Relay Node is Mobile: We assume that the source and the destination nodes are fixed in $[0, L]$, and that the relay node moves along this interval according to either the Random Direction or the Random Waypoint mobility model.

We first focus on the stability condition. From Remark 1 the stability condition is given by $r_{s} \pi_{s}<r_{d} \pi_{d}$, where these quantities are defined in Section III. Let us compute $\pi_{s}$ and $\pi_{d}$ for either mobility model (recall that $r_{s}$ and $r_{d}$ are given parameters). We have

$$
\pi_{s}=\int_{x=(s-R)^{+}}^{(s+R) \wedge L} f(x) d x, \quad \pi_{d}=\int_{x=(d-R)^{+}}^{(d+R) \wedge L} f(x) d x,
$$

where $f(\cdot)$ is the stationary node location distribution, and $a \wedge b=\min (a, b)$. Thus, the stability condition reads

$$
r_{s} \int_{x=(s-R)^{+}}^{(s+R) \wedge L} f(x) d x<r_{d} \int_{x=(d-R)^{+}}^{(d+R) \wedge L} f(x) d x .
$$

Consider now the relay throughput. In the stable case it is given by (see Theorem 2)

$$
r_{s} \int_{x=(s-R)^{+}}^{(s+R) \wedge L} f(x) d x
$$

In the particular case where the relay node moves according to the Random Direction mobility model, the stability condition is (use (26)) with $f(x)$ given in (25))

$$
r_{s}((s+R) \wedge L)-(s-R)^{+}<r_{d}((s+R) \wedge L)-(s-R)^{+},
$$

and the relay throughput, $T_{R D}^{f}$, achieved is (cf. (25) and (27))

$$
T_{R D}^{f}=r_{s} \frac{((s+R) \wedge L)-(s-R)^{+}}{L} .
$$

For $R<s<L-R$ and $R<d<L-R, T_{R D}^{f}=r_{s} \frac{2 R}{L}$ and $\pi_{s}=\pi_{d}$, regardless of the position of the source node and of the destination node. In this case, the stability condition reduces to $r_{s}<r_{d}$.

When the relay moves according to the Random Waypoint mobility model, then the stability condition is (use (24) and (26))

$$
\begin{aligned}
& r_{s}\left[2(A-B)\left(3-\left(A^{2}+A B+B^{2}\right)\right)\right] \\
< & r_{d}\left[2(C-D)\left(3-\left(C^{2}+C D+D^{2}\right)\right)\right],
\end{aligned}
$$


with

$$
\begin{array}{ll}
A:=(s+R) \wedge L, & C:=(d+R) \wedge L, \\
B:=(s-R)^{+}, & D:=(d-R)^{+},
\end{array}
$$

and the relay throughput, $T_{R W}^{f}$, is given by

$$
T_{R W}^{f}=r_{s} \frac{2(A-B)\left(3-\left(A^{2}+A B+B^{2}\right)\right)}{L^{3}} .
$$

2) All Nodes are Mobile: We now assume that the source, destination and relay nodes are all mobile, and move along $[0, L]$ according to the same mobility model: the Random Waypoint or the Random Direction model. First, observe from Remark 2, that in this case the stability condition is given by $r_{s}<r_{d}$.

Let us now compute the throughput $T_{r}=r_{s} \pi_{s}$ for each mobility model. We have

$$
\begin{gathered}
\pi_{s}=\int_{0}^{L} f(x) \int_{(x-R)^{+}}^{(x+R) \wedge L} f(y) d y d x-\int_{0}^{L} f(x)\left[\int_{(x-R)^{+}}^{(x+R) \wedge L} f(y) d y\right]^{2} d x \\
-\int_{0}^{L} f(x) \int_{(x-R)^{+}}^{(x+R) \wedge L} f(y) \int_{(y-R)^{+}}^{(y+R) \wedge L} f(z) d z d y d x
\end{gathered}
$$

where $f(x)$ is given either by (24) or by (25), depending on the mobility model in use. It is easy to compute $\pi_{s}$ in explicit form for both functions $f(x)$. We will instead provide compact approximation formulas, since the exact ones are lengthy. We approximate $\pi_{s}$ by the first term in the r.h.s. of (30). Note that this term is the probability that two nodes are neighbors. This approximation is justified by the fact that, for each function $f(x)$ in (24) and (25), the second and the third term in the r.h.s. of (30) are much smaller than the first term, when the ratio $\rho:=R / L$ is small with respect to 1 . This yields the following approximate throughputs:

$$
T_{R W} \approx r_{s} \frac{\rho\left(12-20 \rho^{2}+15 \rho^{3}-2 \rho^{5}\right)}{5}
$$

for the Random Waypoint mobility model, and

$$
T_{R D} \approx r_{s} \rho(2-\rho),
$$

for the Random Direction mobility model. Observe that these formulas depend on $R$ and $L$ only through their ratio, and that $T_{R D}<T_{R W}$ for all $\rho<1$.

We conclude this section by considering the case where $r_{s}=r_{d}:=r$. In this case, the relay buffer is not stable. To handle this situation, it is proposed in [9] to use a probability of relaying, $p_{r}$, which is close to 1 , so that when the relay node enters the neighborhood of the source node, the source node transmits data to be relayed with probability $p_{r}<1$, and does not transmit with the complementary probability. Note that this scheme ensures stability and gives near maximum throughput as well.

\section{B. Two Dimensions}

In this section we consider nodes moving in a square. We start by computing the relay throughput; then we find the probability of these nodes form a two-hop route.
1) Three Nodes Moving: Nodes move independently of each other inside a square of side length $L$. They move according to the same mobility model, the Random Direction or the Random Waypoint model.

Similarly to Section V-A.2 the stability condition is $r_{s}<r_{d}$ (Remark 2). The probability that two nodes are neighbors is

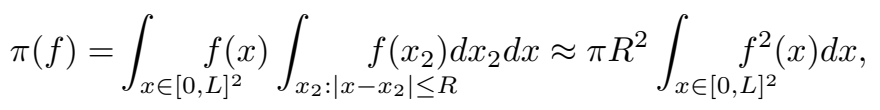

where we have used the continuity of $f(\cdot)$, and the assumption that $R$ is negligible with respect to $L$. This approximation is in agreement with Theorem 3, which states that the minimum probability of contact is achieved by the uniform distribution, since the latter integral is the $L_{2}$-norm of $f(\cdot)$, which is minimized when $f(\cdot)$ is the uniform distribution.

When the RB is stable, the relay throughput is approximated by $r_{s} \pi(f)$, where $r_{s}$ is the source transmission range. It can be shown, numerically, that for the Random Waypoint mobility model over a square $\pi(f) \approx 1.36 \pi(R / L)^{2}$ [7], this implies that the relay throughput, $T_{R W}^{2 d}$, is approximated by

$$
T_{R W}^{2 d} \approx 1.36 \pi r_{s} \rho^{2},
$$

where $\rho:=R / L$. Under the Random Direction mobility we find that $\pi(f) \approx \pi \rho^{2}$. Hence, the relay throughput, $T_{R D}^{2 d}$, is approximated by

$$
T_{R D}^{2 d} \approx \pi r_{s} \rho^{2} .
$$

Note that $T_{R D}^{2 d}<T_{R W}^{2 d}$.

2) Probability of a Two-Hop Route: The throughput of data between a pair of nodes when there exists a route between them, called forwarding throughput, is a function of the route length in hops. A first step to derive the forwarding throughput is to compute the distribution of the route length in hops. In this section, we compute the probability of a two-hop route between two nodes $s$ and $d$, assuming there are $N$ other nodes. All the nodes are mobile inside a square $A=[0, L]^{2}$ and moving (independently) according to either the Random Waypoint or Random Direction models.

Let $x_{s}$ (resp. $x_{d}$ ) represents the position of node $s$ (resp. $d$ ). There is a two-hops route between node $s$ and $d$, if the node $d$ is inside the annulus, $C$, of center $x_{s}$ and of interior and exterior radius equal to $R$ and $2 R$, and there is at least an intermediate node inside, $D_{I}$, the intersection of the two disks of radius $R$ centered around $x_{s}$ and $x_{d}$. The probability of the two-hop routes between nodes $s$ and $d$ when $R<<L$ writes

$$
\begin{aligned}
P^{N}(2) & \approx \int_{A} f\left(x_{s}\right) \int_{C} f\left(x_{d}\right)\left[1-\overline{\left(\int_{D_{I}} f(x) d x\right)^{N}}\right] d x_{d} d x_{s} \\
& \approx 2 \pi \sum_{i=1}^{N}(-1)^{i+1}\left(\begin{array}{c}
N \\
i
\end{array}\right) u(i) v_{f}(i)\left(\frac{R}{L}\right)^{2(i+1)}
\end{aligned}
$$

where $u(i)=\int_{1}^{2} r A(r)^{i} d r$, and $v_{f}(i)=\int_{[0,1]^{2}} f(x)^{i+2} d x$. Here $A(r)$ is the area of $D_{I}$ when $R=1$ and the distance between nodes $s$ and $d$ is equal to $r$. This gives

$$
A(r)=2 \operatorname{arcos}\left(\frac{r}{2}\right)-\frac{r}{2} \sqrt{4-r^{2}} .
$$


We observe that $P^{N}(2)$ is a function of $(R / L)^{2}$. The numerical values of $u(i)$ and $v_{f w}(i)$, the value of $v_{f}(i)$ for the Random Waypoint model, are listed in Table I. Note that for the Random Direction model the node location distribution is uniform over $A$, hence $v_{f d}(i)$, the value of $v_{f}(i)$ in such case, is equal to one.

\begin{tabular}{|c|c|c||c|c|c|}
\hline $\mathbf{i}$ & $u(i)$ & $v_{f w}(i)$ & $\mathbf{i}$ & $u(i)$ & $v_{f w}(i)$ \\
\hline \hline 1 & 0.649 & 2.156 & 8 & 0.4513 & 170.9 \\
\hline 2 & 0.47 & 3.664 & 9 & 0.494 & 341.7 \\
\hline 3 & 0.406 & 6.541 & 10 & 0.547 & 688.8 \\
\hline 4 & 0.384 & 12.08 & 20 & 2.159 & $9.9310^{5}$ \\
\hline 5 & 0.383 & 22.87 & 40 & 66.23 & $3.6810^{12}$ \\
\hline 6 & 0.395 & 44.1 & 60 & 2703.08 & $3.8010^{18}$ \\
\hline 7 & 0.418 & 86.3 & 120 & $3.0910^{8}$ & $7.0910^{38}$ \\
\hline
\end{tabular}

TABLE I

THE NUMERICAL VALUES OF THE $u(i)$ AND $v_{f}(i)$ FOR THE RANDOM WAYPOINT MODEL.

Until now we have looked at the effect of mobility patterns on the "average" throughput and showed that throughput depends only on the stationary node distribution. We then showed that minimum throughput is achieved when the stationary distribution of node position is uniform. In the next section, we will study the relay buffer behavior in the case where the relay node performs a Random Walk with fixed source and destination, and in the case all the three nodes move according to Random Direction model inside a square.

\section{RELAY BUfFER BEHAVIOR}

In this section, the effect of the mobility model on the relay buffer occupancy is studied. We assume that the mobility models under consideration have stationary node location distributions. The plan is to use the $G / G / 1$ queueing model of the RB size introduced in Section III-A, and to look at the effect of mobility patterns on the relay buffer occupancy.

More precisely, in Equation (3) the RB size at the beginning of $n^{t h}$ cycle, $\tilde{B}_{n}$, was interpreted as the workload seen by the $(n+1)^{s t}$ arrival in a $G / G / 1$. In this section, the distribution of $\tilde{B}_{n}$ will be approximated by the corresponding quantity in a $G I / G / 1$, i.e., by assuming that $\alpha_{n}$ and $\sigma_{n}$ in (3) are independent. In the $G I / G / 1$ queue, it is known that $E[\tilde{B}]$ verifies the following inequality called the Kingman upper bound [13, P. 29]

$$
E[\tilde{B}] \leq \frac{r_{d}^{2} \operatorname{Var}\left(\alpha_{n}\right)+r_{s}^{2} \operatorname{Var}\left(\sigma_{n}\right)}{2\left(r_{d} E\left[\alpha_{n}\right]-r_{s} E\left[\sigma_{n}\right]\right)},
$$

and furthermore in the heavy traffic case, i.e., when $r_{s} E\left[\sigma_{n}\right] \approx$ $r_{d} E\left[\alpha_{n}\right]$ with $r_{s} E\left[\sigma_{n}\right]<r_{d} E\left[\alpha_{n}\right]$, the stationary waiting time is exponentially distributed with mean [13, P. 29]

$$
E[\tilde{B}] \approx \frac{r_{d}^{2} \operatorname{Var}\left(\alpha_{n}\right)+r_{s}^{2} \operatorname{Var}\left(\sigma_{n}\right)}{2\left(r_{d} E\left[\alpha_{n}\right]-r_{s} E\left[\sigma_{n}\right]\right)} .
$$

This approximation will be used in the following to estimate the expected RB size for the following cases where: first the relay node performs a Random walk and the source and destination are fixed, second the three nodes move inside a square according to the Random Direction model. This section is meant for illustration of the above phenomenon.
Moreover, it is also to be understood that the effective arrival process to the RB in the $G / G / 1$ queueing model is not the contact time between the relay and source nodes, i.e.,

$$
\int_{u=Z_{n}}^{Z_{n+1}} \mathbf{1}_{\{S(u)=1\}} d u,
$$

but is composed of many (random number of) such contact times since

$$
\sigma_{n}=\int_{u=W_{n}}^{W_{n+1}} \mathbf{1}_{\{S(u)=1\}} d u .
$$

That this is the case can be easily seen by studying the evolution of the $\left\{S_{t}\right\}$ process. Since it is possible that in a cycle, the $\left\{S_{t}\right\}$ process alternates between values 0 and 1 for many times before taking the value of -1 .

Clearly, a larger relay buffer occupancy would imply that the amount of time required to deliver all the packets would be composed of many contact periods between the relay node and the destination, hence there can be several inter-visits between the relay node and the destination required to deliver the packets. This implies that we can not study the delay incurred by the nodes by considering only one inter-visit time (or the meeting time) or only one contact time. This shows that the buffer behavior (hence the delays) will depend on both contact times and the inter-visit times.

This section is meant for illustration of the above phenomenon.

\section{A. One-dimensional Random Walk}

We consider the following scenario. The relay node is moving according to a symmetric random walk (RW) on a circle of circumference ${ }^{1} 4 R+2 w$ steps - see Figure 2 . The RW step size is fixed and is equal to $\mu$ meters. The speed of the relay node is assumed to be constant and equal to $v$, so the time required to jump from one step to the next one, is equal to $\mu / v$ seconds. The source and the destination are held fixed, and they are located as shown in Figure 2. The quantities $w$ and $R$ are assumed to be integers. Also, the data transmission between source and destination only takes place through the relay node.

When the relay node becomes a neighbor of the source (when passing points $\mathrm{E}$ or $\mathrm{F}$ ), it starts to accumulate data at rate $r_{s}$. When the relay node enters the neighborhood of the destination, via points $\mathrm{G}$ or $\mathrm{H}$, it delivers the data to destination at rate $r_{d}$. Once in the interval $[E, F]$, the relay node remains there for a random amount of time before exiting via points $E$ or $F$. Symmetry implies that this time has the same distribution whether the relay node enters $[E, F]$ through the point $E$ or $F$. Similar is the case for the segment $[G, H]$. We call this (random) time the contact time between the relay node and the source (or the destination). Once the relay node exits $[E, F]$, it either enters $[J, K]$ or $[I, O]$. Now, the relay node stays in this region for a random amount of time (during which it neither receives nor transmits), and then either reenters $[E, F]$ or enters $[G, H]$.

\footnotetext{
${ }^{1} R$ is the transmission range of source, destination, and relay node.
} 


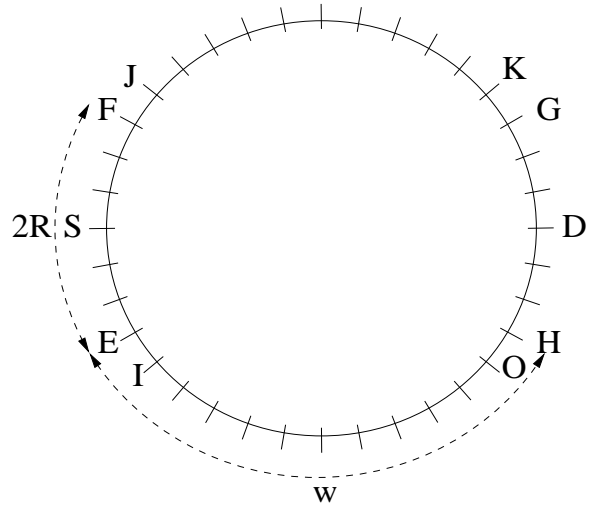

Fig. 2. Random walk on circle.

The number of times that the relay node enters $[E, F]$ without entering $[G, H]$ is denoted by the r.v. $L$, and is geometrically distributed with parameter $p$, independent of whether the relay node exited $[E, F]$ via $E$ or $F$, that is,

$$
P(L=k)=(1-p) p^{k-1} .
$$

The parameter $p$ is the probability that a symmetric random walker starting at point $J$ hits point $F$ before reaching $G$.

Let $A_{j}, j \geq 1$, be independent and identically distributed random variables representing the first time that a random walker, starting at point $F$, exits $[E, F]$, so that the service requirement in the queueing model of Section III-A is $r_{s} \sigma$, where

$$
\sigma=\sum_{j=1}^{L} A_{j}
$$

In the following, $A$ denotes a generic r.v. with the same distribution as $A_{j}$.

Using results from random walk literature (for example [6]), it can be shown that

$$
\begin{aligned}
E[A] & =2 R \frac{\mu}{v} \\
\operatorname{Var}[A] & =\left(\frac{\mu}{v}\right)^{2} \cdot \frac{4 R}{3}(2 R+1)(R+1), \\
p & =1-\frac{1}{w} \\
E[L] & =w \\
\operatorname{Var}[L] & =w(w-1) .
\end{aligned}
$$

Since $L$ is independent of $A$, we get

$$
\begin{aligned}
E[\sigma] & =E[A] E[L]=2 w R \frac{\mu}{v}, \\
\operatorname{Var}[\sigma] & =\operatorname{Var}[A] E[L]+(E[A])^{2} \operatorname{Var}[L], \\
& =4 w R\left(\frac{\mu}{v}\right)^{2}\left(w R+\frac{1}{3}\left(2 R^{2}+1\right)\right) .
\end{aligned}
$$

In the scenario under study, $\sigma_{n}$ and $\alpha_{n}$ have the same probability distribution (Remark 2). Thus based on (38), the expected RB size at the beginning of the cycles is approximately exponentially distributed with expected value

$$
E[\tilde{B}]=\frac{\left(r_{s}^{2}+r_{d}^{2}\right) \operatorname{Var}(\sigma)}{2 E[\sigma]\left(r_{d}-r_{s}\right)}=\frac{\mu\left(r_{s}^{2}+r_{d}^{2}\right)}{v\left(r_{d}-r_{s}\right)}\left(w R+\frac{1}{3}\left(2 R^{2}+1\right)\right),
$$

when $r_{s} \approx r_{d}$ with $r_{s}<r_{d}$.

\section{B. Random Direction Mobility on a Plane}

We consider the following scenario of three nodes: the source, the destination, and the relay node. All these nodes are moving inside a square of side-length $L$. They move according to the Random Direction model with wraparound and with constant speed $V$, and constant travel time $T$.

Based on the queueing model of Section III-A, the RB accumulates data at rate $r_{s}$ when the process $S_{t}=1$. This means that the relay node is inside the transmission range of the source, in contact with the source, and the destination is outside the transmission range of the source and of the relay node. In the first hand, note that when $R<<L$ the probability that the source and the relay node are in contact is of order $(R / L)^{2}$, see Section V-B.1. But on the other hand, the probability that the relay node and the destination are in contact with the source is of order $(R / L)^{4}$, the same order as the probability that the three nodes form a two hop route, see Section V-B.2. Thus, we will approximate the duration of time where $S_{t}=1$ by the duration of time where the source and the relay node are in contact regardless of the position of the destination.

Let $A_{j}, j \geq 1$, be independent and identically distributed random variables representing the contact times between the source and the relay node. Recall that in Section III-A a cycle contains at least one contact time between source and relay node and one contact time between relay node and destination. Let $L_{1}$ denotes the r.v. that represents the number of times that a contact is established between the source and relay node inside a cycle. The service requirement in the queueing model of Section III-A is $r_{s} \sigma$, where

$$
\sigma=\sum_{j=1}^{L_{1}} A_{j}
$$

As we saw in Section VI-A, the mean RB size at cycle instants in heavy-traffic depends on the first two moments of $L_{1}$ and $A_{j}$. Thus in the rest of the section, first we will derive the probability distribution of $L_{1}$, and next we will derive the probability distribution of $A_{j}$ using that of $L_{1}$.

For $i \geq 1$, let $I_{i}$ denote the inter-meeting time between the source and the relay node, i.e. the time interval between two consecutive contacts of the two nodes. Let $F_{r}$ denote the residual time (from start of a cycle) required for the relay node and the destination to come in contact with each other. We will assume that $I_{i}, A_{i}$, and $F_{r}$ are mutually independent [11]. For $k \geq 1$, let $X_{k}=\sum_{i=1}^{k}\left(A_{i}+I_{i}\right)$ and $X_{0} \triangleq 0$, so the probability that $L_{1} \geq k$ given that $L_{1} \geq 1$ is

$$
P\left(L_{1} \geq k\right)=\frac{P\left(X_{k-1}+A_{k} \leq F_{r}\right)}{P\left(A_{1} \leq F_{r}\right)} .
$$

For $R<<L$, the inter-meeting times between any pair of nodes, $I_{i}$, are well approximated by independent and identically distributed r.v. of exponential distribution with parameter $\lambda=\frac{8 R V}{\pi L^{2}}$ [8]. Then, using the memoryless property of the exponential distribution, it follows that $F_{r}$ is also exponentially 


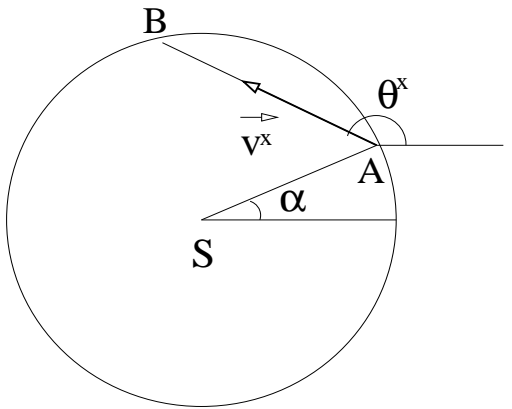

Fig. 3. Contact distance between 2 mobile nodes.

distributed with the same parameter $\lambda$. Therefore, in our case the probability $P\left(L_{1} \geq k\right)$ is approximated by

$$
P\left(L_{1} \geq k\right) \approx \beta^{k-1},
$$

where $\beta=\frac{1}{2} \exp (-\lambda E[A])$, with $E[A]$ denoting the average value of the r.v. $A_{j}$. The above approximation is based on the assumption that variation of $A_{j}$ is small compared to those of $X_{k}$ and $F_{r}$. In the rest of the section, we will derive the probability distribution of $A_{j}$.

Let $A$ denotes the r.v. that have same distribution as $A_{j}$. Assume that during the contact of two nodes there is no change of node direction. This assumption holds when $T$, the node travel time between successive change of directions, is sufficiently large compared to the contact time between two nodes. By conditioning on the direction of the source, $\theta_{s}$, and of the relay node, $\theta_{r}$, we derive the relative speed $v^{*}=\left|\vec{V}\left(\theta_{s}\right)-\vec{V}\left(\theta_{r}\right)\right|$ and the relative angle $\theta^{*}=\theta_{s}-\theta_{r}$ between these two nodes. The crossing distance, $A B$, depends on the crossing angle $\alpha$, see Figure 3. By conditioning on $\alpha, A$ is computed by dividing $A B$ by the relative speed $v^{*}$. We note that in the case of the Random Direction model $\theta_{s}, \theta_{r}$, and $\alpha$ are uniform distributed in $[0,2 \pi]$. The CDF of $A$ is computed by unconditioning on $\theta_{s}, \theta_{r}$, and $\alpha$, over the constraint that $\left|\theta_{s}-\theta_{r}\right|>\delta$, where $\delta$ is a threshold to avoid very large contact time occurrence. Let $\theta_{1}=\frac{\theta_{s}+\theta_{r}}{2}$, and $\theta_{2}=\frac{\theta_{s}-\theta_{r}}{2}$, thus the CDF of the normalized contact time $\tilde{A}=A \frac{V}{R}$ is the following

$$
\begin{aligned}
& P(\tilde{A} \leq x)=\frac{1}{C}\left[\int_{\delta}^{2 \pi} \int_{0}^{\theta_{s}-\delta} \int_{0}^{\frac{\pi}{2}} d \alpha d \theta_{s} d \theta_{r}+\right. \\
& \int_{0}^{2 \pi-\delta} \int_{\theta_{1}+\delta}^{2 \pi} \int_{0}^{\frac{\pi}{2}} d \alpha d \theta_{s} d \theta_{r}+\int_{\delta}^{2 \pi} \int_{0}^{\theta_{s}-\delta} \int_{\frac{\pi}{2}}^{2 \pi} d \alpha d \theta_{s} d \theta_{r} \\
& \left.+\int_{0}^{2 \pi \leq \theta_{1}-\pi, \sin \left(\theta_{1}-\alpha\right) \geq x \cdot \sin \left(\theta_{2}\right)} \quad \theta_{\theta_{1}+p i \leq \alpha \leq 2 \pi, \sin \left(\theta_{1}-\alpha\right) \leq x \cdot \sin \left(\theta_{2}\right)}^{2 \pi-\delta} \int_{\theta_{s}+\delta}^{2 \pi} \int_{\frac{\pi}{2}}^{2 \pi} d \alpha d \theta_{s} d \theta_{r}\right] \\
& \theta_{1} \leq \alpha \leq \theta_{1}+\pi, \sin \left(\theta_{1}-\alpha\right) \geq x \cdot \sin \left(\theta_{2}\right)
\end{aligned}
$$

where $C$ is a normalization constant. We observe that the CDF of the normalized contact time is independent of $R$ and $V$, and that it is easy to compute numerically the moments of $\tilde{A}$. For example for $\delta=0.1 \pi$, we have that $C=0.3298$, $E[A]=1.1778 \frac{R}{V}$, and $E\left[A^{2}\right]=2.3365\left(\frac{R}{V}\right)^{2}$.

Supposing that $L_{1}$ is independent of $A$, the mean and variance of $\sigma$ are derived. So based on (38), in heavy-traffic the $\mathrm{RB}$ size at the the beginning of the cycles is approximately exponentially distributed with mean value

$$
E[\tilde{B}]=\left(0.403+0.5889 \frac{\beta}{1-\beta}\right) \frac{r_{s}^{2}+r_{d}^{2}}{r_{d}-r_{s}} \frac{R}{V},
$$

for $r_{s} \approx r_{d}$ with $r_{s}<r_{d}$. We observe that $E[\tilde{B}]$ is function of $R$, and $V$ only through the ratio $R / V$. Further in Section VII-H that the event average $E[\tilde{B}]$ converges to, $E\left[B_{t}\right]$, the time average of the RB size in the heavy-traffic.

\section{NUMERICAL RESULTS}

In this section we present simulation results to validate results in Theorem 1 (stability issues), Theorem 2 (throughput depends only on stationary distribution), Section V (throughputs obtained by Random Waypoint and Random Direction Models), the probability of two-hop route, the relay buffer behavior as studied in Section VI, and the probability distribution of the contact time. Throughout this section, we will assume that the transmission range of the nodes is constant and is equal to $R$.

\section{A. Validation of Theorem 1}

We consider the scenario of three nodes: a source, a destination, and a relay node. each moving according to a symmetric random walk over a circle of circumference length equal to $4000 \mathrm{~m}$. It follows from Remark 2 that the relay node buffer occupancy is stable iff the ratio $p=\frac{r_{s}}{r_{d}}<1$. Figure 4 plots the evolution of relay node buffer with time for different values of $p$.

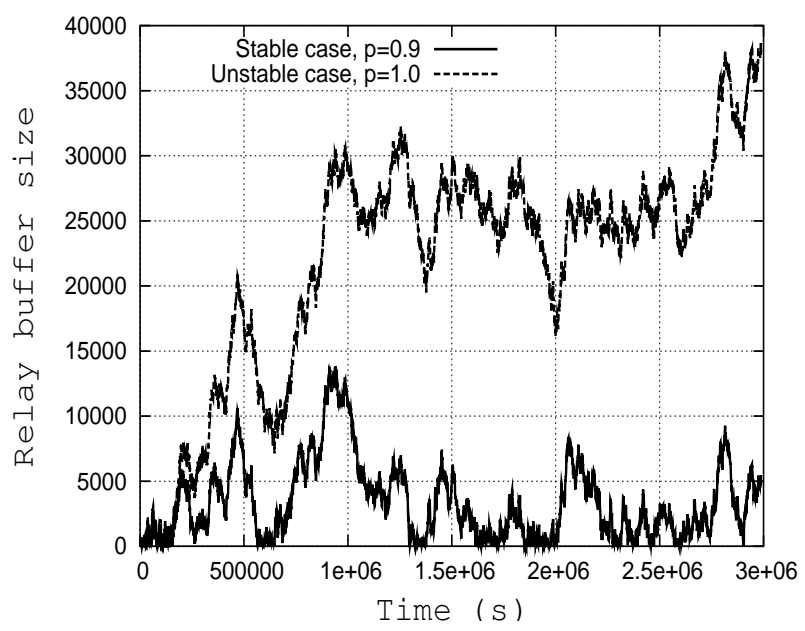

Fig. 4. Time-evolution of relay node buffer for Random Walk model over a circle for different values of ratio, $p=\frac{r_{s}}{r_{d}}$.

It is evident from the figure that when $p=1.0$, the buffer occupancy process is unstable. While for the case $p=0.9<$ 1.0 , this process is stable. Similar results were obtained even for $p \approx 1.0$ with $p<1.0$ but are not shown here.

Figure 5 plots the evolution of the mean relay node buffer as a function the ratio $p$ for Random Direction model inside a square of side-length $1000 \mathrm{~m}$ and for two different values of $R=\{50,100\} m$. The nodes' speed (resp. nodes' travel time) 
is uniformly distributed inside $[5 \mathrm{~m} / \mathrm{s}, 15 \mathrm{~m} / \mathrm{s}]$ (resp. $[9 \mathrm{~s}, 11 \mathrm{~s}]$ ). Observe that the mean relay node buffer size increases rapidly as $p$ approaches one.

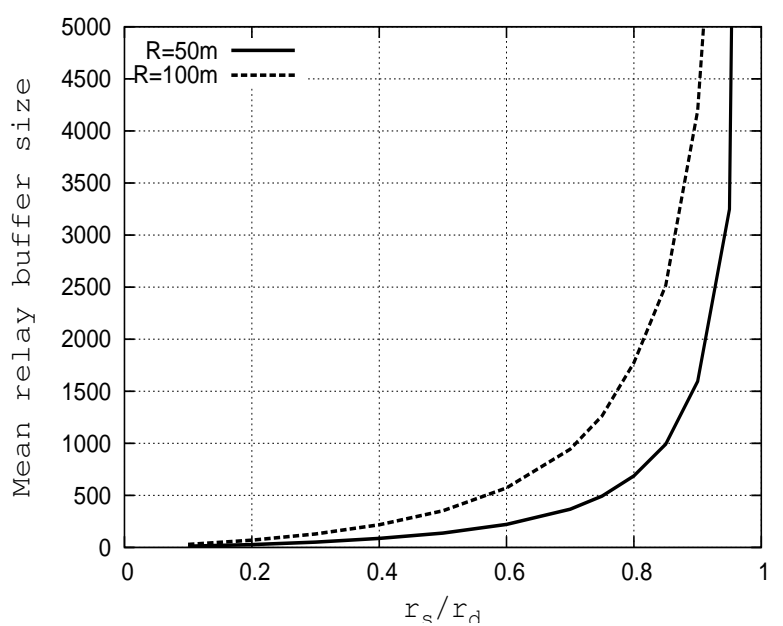

Fig. 5. Mean relay buffer size as a function of the ratio $p=\frac{r_{s}}{r_{d}}$ for Random Direction mobility and for diferent value of $R$

\section{B. Validation of Theorem 2 and Section $V$}

Theorem 2 states that the relay throughput depends only on the stationary node distribution. Section $\mathrm{V}$ provides the value of relay throughput under Random Direction and Random Waypoint mobility models. To validate both of these results, we ran simulations to find the relay throughput for case of the Random Direction and the Random Waypoint mobility models with different parameters.

We illustrate that the throughput depends only on the stationary distribution of the node position by looking at the scenario where three nodes move over a line of length $L=$ $4000 \mathrm{~m}$ according to the Random Direction model. We assume that the time between two consecutive decision instants (travel time) is fixed and equal to 15 seconds and the distribution of speed was chosen to be i) Uniform over some interval, ii) Exponentially distributed, and iii) fixed. Note that the case where speed is fixed corresponds to the Random Walk. Since the stationary node location distribution is same for all the three choice of speed distribution, Theorem 2 implies that the relay throughput will be identical. The numerical results plotted in Figure 6 are in accordance with this result. Also evident is the fact that relay throughput $T_{r}=\pi_{s}$, for $r_{s}=1$. In Figure 7 we plot values of $\pi_{s}$ when the three nodes move according to the Random Waypoint model over a square of side-lengths $L$ for different values of transmission range, $R$. We keep the speed of the mobiles fixed. The plot shows that $\pi_{s}$ (and hence $T_{r}$ ) is a function of $\rho=\frac{R}{L}$ alone. The numerical values also support the result of Section V-B where for the Random Waypoint model in square, the throughput is approximately $1.36 \pi \rho^{2}$. Similarly, the values of the throughput from theory and simulations provide a good match for all of the scenario studied in Section V. Because of the space restriction, we did not include these numerical results due to space constraints.

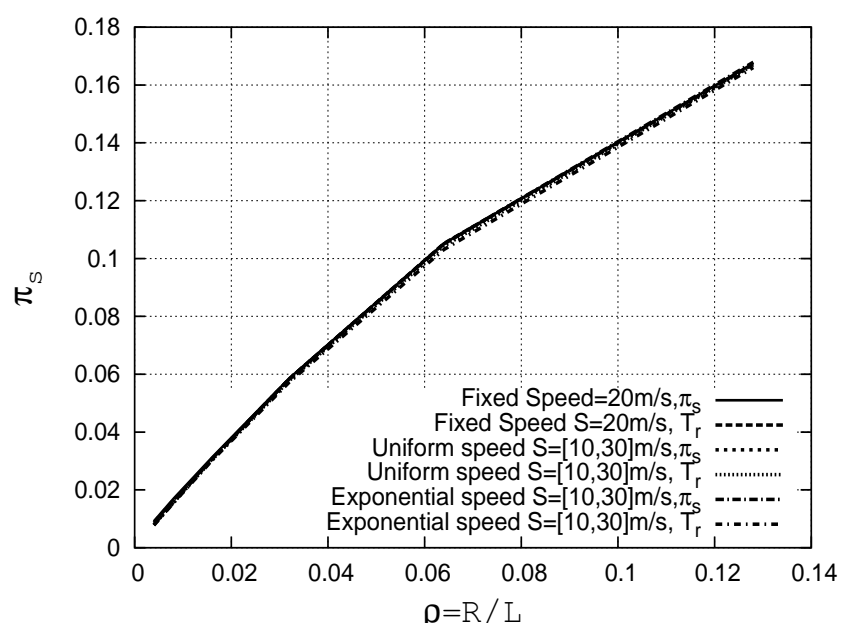

Fig. 6. Values of $\pi_{s}$ obtained for the Random Direction model over a segment of length $L$. Various distributions for the speed were taken.

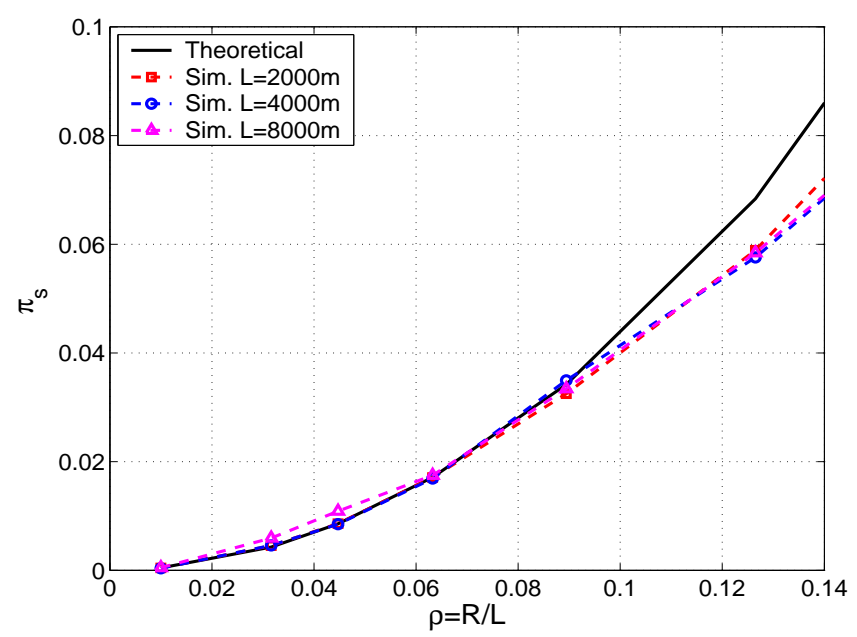

Fig. 7. Simulation results showing $\pi_{s}$ for Random Waypoint model over a square of side-lengths $L$ for different values of transmission range, $R$. Also shown are corresponding values from Section V-B.

\section{Validation of Section III-B}

Section III-B studies multiple relay nodes with fixed source nodes and destination nodes. It reports stability condition and derives the value of the relay throughput and the network throughput. To validate the stability condition, we take the scenario of one source node, one destination node, and 3 relay nodes move according to the Random Waypoint model inside a square of side-length $L=4000 \mathrm{~m}$. The source and destination nodes are fixed and they are symmetric according to the center of the square, and the separated distance between them is of $2000 \mathrm{~m}$. The stable case is shown in Figure 8 where $r_{s}=0.9 r_{d}$, and the unstable case is shown in Figure 9 where $r_{s}=1.1 r_{d}$. The network throughput as a function of the number of the relay nodes are shown in Figure 10 for different value of $R / L$. In this scenario, the probability that the relay node is neighbor of the source of location $(1000,1000)$ is equal to $0.0485,0.0275$, and 0.0126 for $R / L$ equal to 0.1 , 0.075 , and 0.05 respectively. 


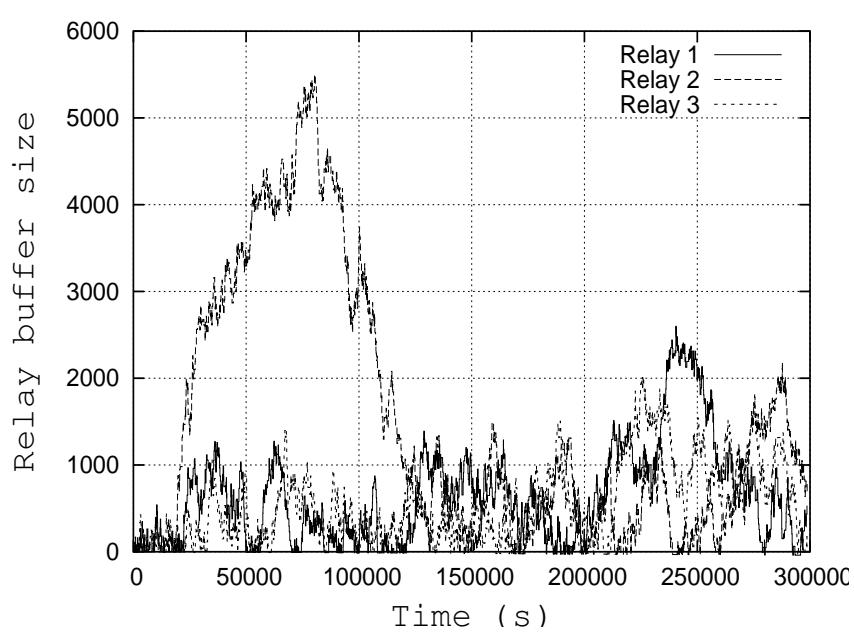

Fig. 8. Time-evolution of relay buffer for three relay nodes moving according to Random Waypoint model inside a square of side-length $4000 \mathrm{~m}$, with fixed and symmetric source and destination node w.r.t. to square center. Here $r_{s}=$ $0.9 r_{d}$ (the stable case)

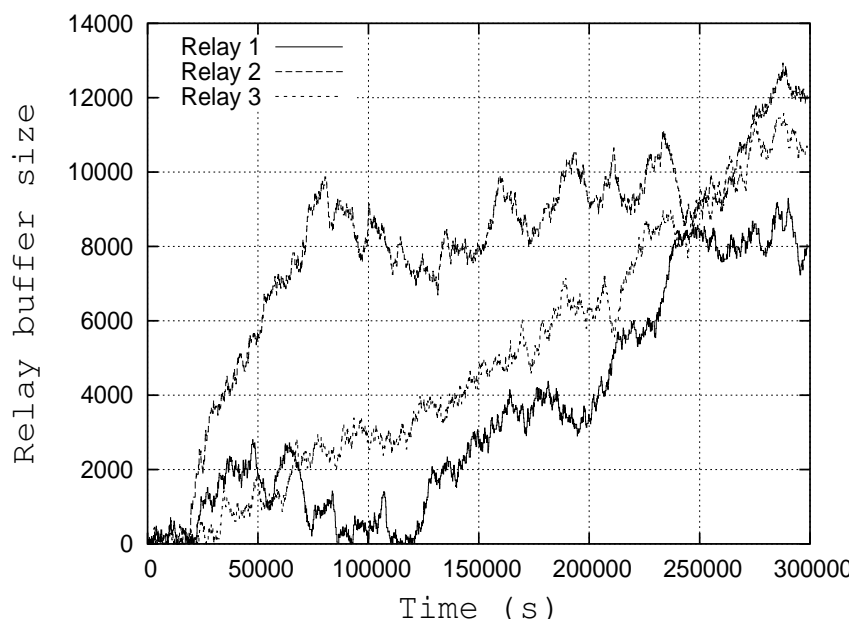

Fig. 9. Time-evolution of relay buffer for relay nodes moving according to Random Waypoint model inside a square of side-length $4000 \mathrm{~m}$, with fixed and symmetric source and destination nodes w.r.t.to square center. Here $r_{s}=$ $1.1 r_{d}$ (the unstable case)

\section{Validation of Section III-B}

In this section we validate the approximation of Section III-B of the case where all source and destination nodes are moving (c.f, Equation (16)). We consider a scenario of $N$ relay nodes and $K$ source nodes and $M$ destination nodes. All the nodes move according to the Random Waypoint model within a square region of side-length $L$ equal to $4000 \mathrm{~m}$. We assume that the lifetime of the packets in hops is equal to 4 hops. In Figure 11, we show the approximation (c.f, Equation (16)) as well as the simulation result of, $\tau_{S}^{m}$, the long-term arrival rate to the queue $m$ of the relay node $n$ from the source nodes. We observe that the above approximation is accurate for $R / L \leq$ 0.05 , and the relative error between the approximation and simulation is less than $5 \%$, for $R / L=0.05$.

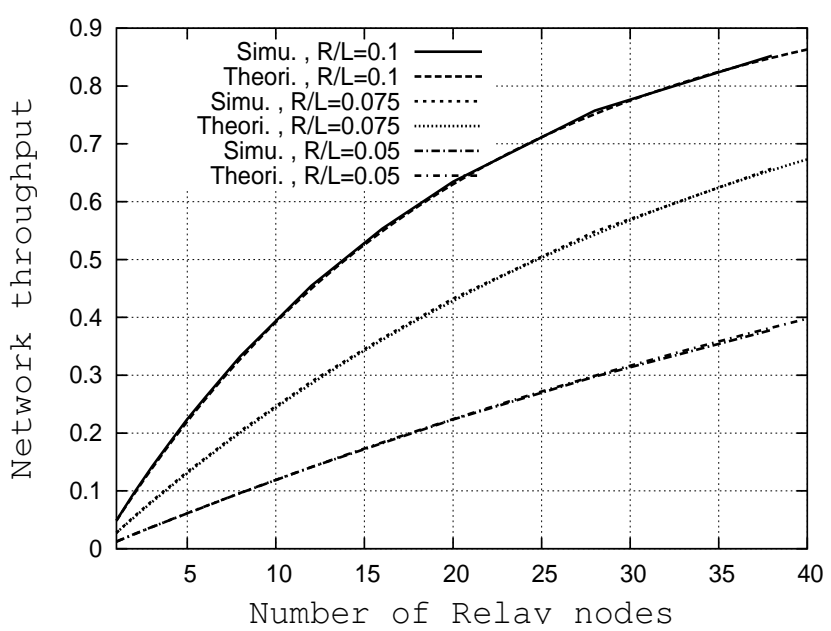

Fig. 10. Network throughput as function of number of relay nodes in square region of side-length $4000 m$ with fixed source and fixed destination nodes.

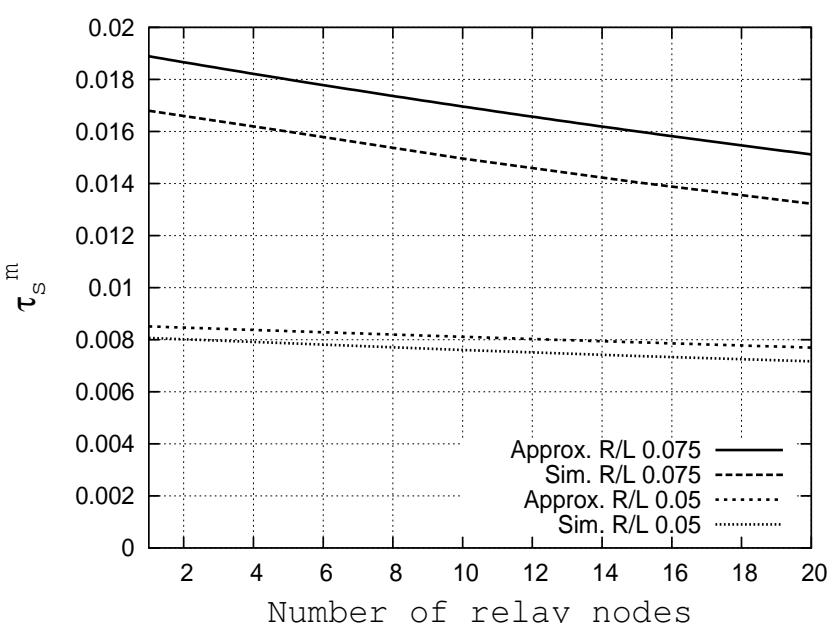

Fig. 11. Long-term arrival rate at queue $m$ of relay node $n$ from source node $k$ as function of number of relay nodes $N$, where $K=4, M=5$, $P_{k}^{m}=1 / M$, and $r_{S_{k}}=1$.

\section{E. Validation of Two-Hop Route Probability}

We have $N+2$ nodes moving inside a square of side length $L=4000 m$ according to the Random Waypoint model. We validate the approximation formula for the probability of a two hops route for different values of $N$ (cf., section V-B.2). In Figure 12, we show the results of the simulation and the approximation for $R / L \in\{0.025,0.0375,0.05\}$. We observe that for $R / L \leq 0.05$ the approximation is accurate.

\section{F. Validation of Section VI-A}

We consider the relay node buffer occupancy in the scenario in Section VI-A. Figure 13 plots the percentage of the relative error of the relay buffer occupancy found in (41) and the corresponding simulated value $E\left[B_{\text {sim }}\right]$ as a function of the load $\frac{r_{s}}{r_{d}}$ and for different values of the parameters $R$ and $w$ with $r_{d}=1, \mu=100 \mathrm{~m}$, and $v=10 \mathrm{~m} / \mathrm{s}$. Parameters $R$ and $w$ are chosen so that the circumference of the circle is equal to 3000 meters (i.e. $(4 R+2 w) \mu=3000 m)$. In the simulation the relay node buffer is sampled at the beginning of each cycle (as 


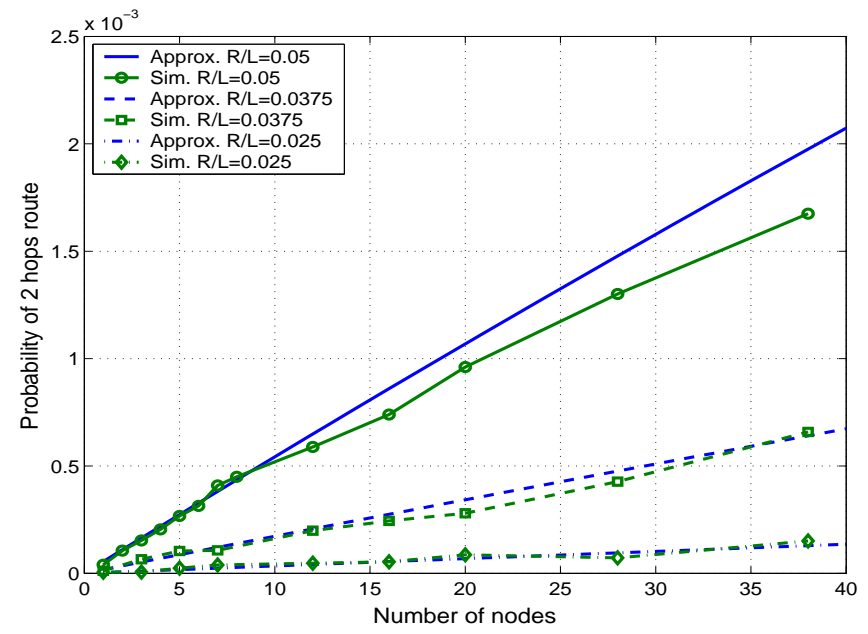

Fig. 12. Probability of two-hop route as function of number of nodes. All nodes move according to Random Waypoint model inside square of side length $4000 \mathrm{~m}$.

defined in Section III). Throughout these experiments, when $\frac{r_{s}}{r_{d}}>0.9$, so as to reflect the heavy-traffic scenario under which (41) was established. We observe that this relative error is almost independent of $R$ and $w$. Furthermore, for $\frac{r_{s}}{r_{d}} \geq 0.95$ the relative error between (41) and the simulation is smaller than $8 \%$.

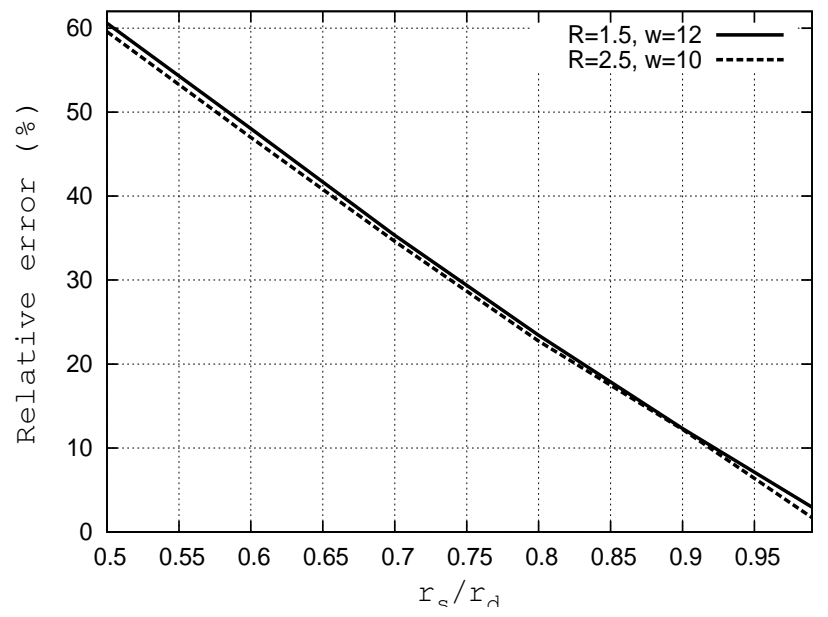

Fig. 13. Percentage of relative error between model in (41) and simulation as a function of $r_{s} / r_{d}$ for different values of $R$ and $w$ and for $r_{d}=1$, $\mu=100 \mathrm{~m}$, and $v=10 \mathrm{~m} / \mathrm{s}$.

\section{G. Validation of Section VI-B}

We consider the scenario where the nodes move according to the Random Direction model inside a square of side length $L=4000 \mathrm{~m}$. The nodes speed is constant and is equal to $V$. The node travel time, $T$, is constant and is greater than $R / V$. First, we validate the approximation of, $\tilde{A}$, the normalized contact time, and next the approximation of the mean buffer size $E[\tilde{B}]$ at cycle time. In Figure 14, we compare the normalized contact time distribution of the simulations for different values of $R / V$ and $T$ with the model (c.f, Equation (45)) for $\delta=0.1 \pi$. We observe that similarly as the model predict that the $\mathrm{CDF}$ of $\tilde{A}$ is almost independent of $R / V$, of $R / L$, and of $T$. We note that same observations are also valid in the case of the Random Waypoint model, see Figure 15. In Table II, we show the relative error between the average buffer size computed using the model (c.f, Equation (46)) and the simulation as a function of the $R$ and $V$ for the case of Random Direction model and for $\frac{r_{s}}{r_{d}}=0.99$. We observe that the model is accurate for $R / V<10$.

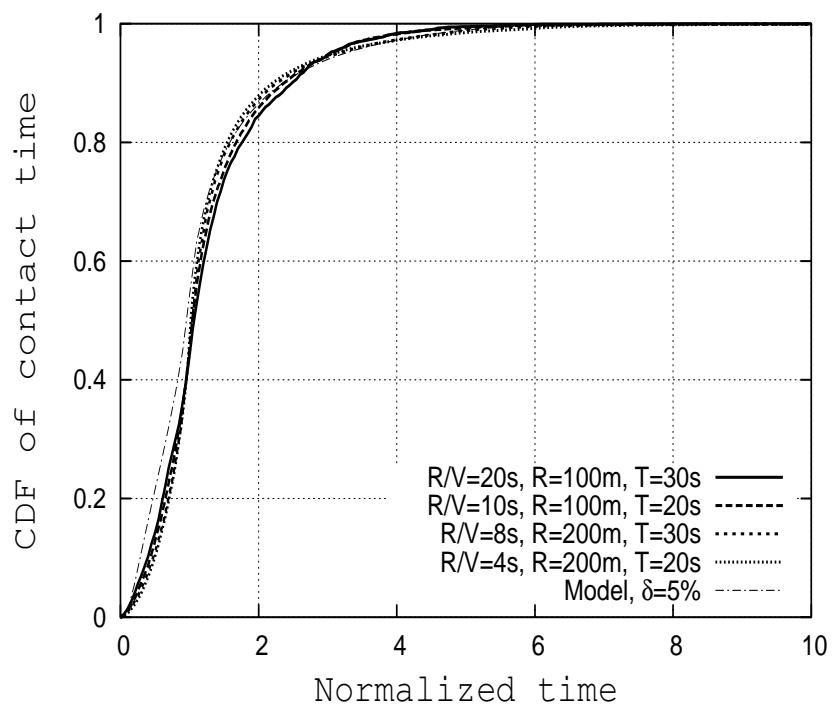

Fig. 14. Normalized contact time distribution for different values of $R / V$ and travel time $T$ comparing with Equation (45). Nodes move according to Random Direction model inside a square of side-length with $L=4000 \mathrm{~m}$.

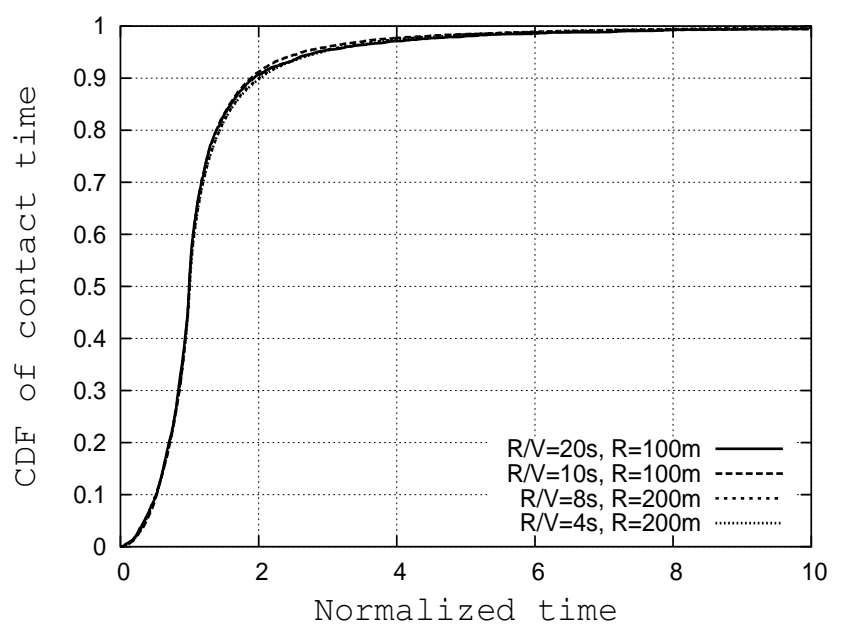

Fig. 15. Normalized contact time distribution. Nodes move according to the Random Waypoint model inside a square of side-length $L=4000 \mathrm{~m}$.

\begin{tabular}{|c|c|c|c|c|}
\hline $\mathbf{R}(\mathbf{m})$ & \multicolumn{2}{|c|}{75} & \multicolumn{2}{c|}{100} \\
\hline $\mathbf{V}(\mathbf{m} / \mathbf{s})$ & 7.5 & 15 & 10 & 20 \\
\hline$\frac{\left|E\left[B_{\text {sim }}\right]-E[\tilde{B}]\right|}{E[\tilde{B}]}$ & 0.14 & 0.03 & 0.14 & 0.05 \\
\hline
\end{tabular}

TABLE II

VALIDATION OF EQUATION (46) FOR $r_{s} / r_{d}=0.99$ AND $r_{d}=10$. 


\section{H. Comparison between $E[\tilde{B}]$ and $E[B]$}

In this section we study the relative difference between, $E[B]$, the RB time average and, $E[\tilde{B}]$, the RB average at the cycle time (event average). We consider the scenario where three nodes move according to the Random Waypoint or to the Random Direction with reflection inside a square of side-length $L=1000 \mathrm{~m}$. For both cases, the nodes speed is uniformly distributed in the interval $[5 \mathrm{~m} / \mathrm{s}, 15 \mathrm{~m} / \mathrm{s}]$. For the Random Direction model the travel time is uniformly distributed inside $[9 s, 11 s]$. Tables III and IV show the relative difference defined as follows $\frac{E[B]-E[\tilde{B}]}{E[B]}$ as a function of $R$, the node's transmission range, and the ratio $\frac{r_{s}}{r_{d}}$. Note that the time average $E[B]$ is computed by sampling the RB size every $0.1 \mathrm{~s}$. The simulation time is considered to be equal to $10^{9} \mathrm{~s}$. We observe that for different values of the $R$ as the $\frac{r_{s}}{r_{d}}$ increases the relative difference decreases and when $\frac{r_{s}}{r_{d}} \stackrel{r_{d}}{=} 0.99$ the relative difference is almost negligible. This means that the event average $E[\tilde{B}]$ is converging to the time average $E[B]$ and these two quantities are almost equal in heavy-traffic. Note that this observation is true for the two mobility models considered.

\begin{tabular}{|c|c|c|c|c|}
\hline \multirow{2}{*}{$\mathbf{R}(\mathbf{m})$} & \multicolumn{4}{|c|}{$r_{s} / r_{d}$} \\
\cline { 2 - 5 } & 0.25 & 0.5 & 0.75 & 0.99 \\
\hline 50 & 0.75 & 0.41 & 0.17 & 0.006 \\
\hline 100 & 0.75 & 0.42 & 0.18 & 0.004 \\
\hline 200 & 0.73 & 0.42 & 0.18 & 0.003 \\
\hline
\end{tabular}

TABLE III

RELATIVE DIFFERENCE BETWEEN MEAN BUFFER SIZE AT $t$ AND MEAN BUFFER SIZE AT CYCLE TIME FOR RANDOM WAYPOINT.

\begin{tabular}{|c|c|c|c|c|}
\hline \multirow{2}{*}{$\mathbf{R}(\mathbf{m})$} & \multicolumn{4}{|c|}{$r_{s} / r_{d}$} \\
\cline { 2 - 5 } & 0.25 & 0.5 & 0.75 & 0.99 \\
\hline 50 & 0.75 & 0.42 & 0.17 & 0.0015 \\
\hline 100 & 0.74 & 0.42 & 0.18 & 0.004 \\
\hline 200 & 0.73 & 0.42 & 0.18 & 0.005 \\
\hline
\end{tabular}

TABLE IV

RELATIVE DIFFERENCE BETWEEN MEAN BUFFER SIZE AT $t$ AND MEAN BUFFER SIZE AT CYCLE TIME FOR RANDOM DIRECTION.

\section{CONCLUSIONS}

We have studied the performance of relaying in mobile ad hoc networks by developing a queueing model. The parameters of the queueing model depend on the node mobility pattern.

Our main findings are that (under the assumptions placed on our model) the relay throughput depends only on the stationary node location distribution, and that uniform stationary distribution of nodes results in the smallest relay throughput. Approximate throughput formulas have been derived for both the Random Waypoint and the Random Direction mobility models; these formulas have been found to be in agreement with simulation results. Approximation formula for the mean buffer occupancy of the relay node has been obtained for the Random Walk mobility model and the Random Direction in both one and two dimensional movement.
We have implicitly assumed that the order of delivery of data does not matter. This is an important simplifying assumption that has allowed us to assume that the relay node does not transmit data from its relay buffer when there is either a direct or two-hop route (via the relay node) between the source and destination. Even though this is a realistic assumption for the delay tolerant networks, relaxing this assumption would be important for applications using TCP-like protocols.

Most of our work in this paper has focused on the relay throughput and average behavior of the relay node buffer occupancy in the abscence of any interference between nodes. It would be more realistic to study the impact of considering nodes' inteference on both throughput and relay buffer size in our future work.

This study forms a research effort towards developing performance models for relay protocol, and understanding the impact of mobility on their performance.

\section{REFERENCES}

[1] Delay Tolerant Research Group, http://www.dtnrg.org.

[2] A. Al Hanbali, A. A. Kherani, R. Groenevelt, P. Nain, and E. Altman. Impact of Mobility on the Performance of Relaying in Ad hoc Networks. Proc. of INFOCOM 2006, Bracelona, Spain, 2006.

[3] F. Baccelli, P. Brémaud. Palm Probabilities and Stationary Queues, Springer-Verlag, 1987.

[4] C. Bettstetter, H. Hartenstein, and X. Prez-Costa. Stochastic Properties of the Random Waypoint Mobility Model. ACM/Kluwer Wireless Networks, Special Issue on Modeling and Analysis of Mobile Networks, vol. 10, no. 5, pp. 555-567, Sept 2004.

[5] A. El Gamal, J. Mammen, B. Prabhakar and D. Shah. Throughput-Delay Trade-off in Wireless Networks Proc. of INFOCOM 2004, Hong Kong, 2004.

[6] W. Feller. Probability Theory and its Applications, vol. 1 New York: John Wiley and Sons, Inc., third ed., 1968.

[7] R. Groenevelt. Stochastic Models in Mobile Ad Hoc Networks Ph.D. Thesis, University of Nice Sophia Antipolis, April 2005.

[8] R. Groenevelt, P. Nain and G. Koole, The Message Delay in Mobile Ad Hoc Networks, Proc. of Performance 2005, Juan-les-Pins, France, October 2005. Published in Performance Evaluation, Vol. 62, Issues 1-4, October 2005, pp. 210-228.

[9] M. Grossglauser and D. Tse. Mobility Increases the Capacity of Ad hoc Wireless Networks IEEE/ACM Transactions on Networking, vol. 10, no. 4, August, 2002, pp. 477-486.

[10] P. Gupta and P. R. Kumar. The Capacity of Wireless Networks IEEE/ACM Transactions on Information Theory, vol. 46, no. 2, March, 2000.

[11] Y. Han, R. J. La, A. M. Makowski, and S. Lee. Distribution of path durations in mobile ad-hoc networks - Palm's Theorem to the rescue To appear in the Special Issue of Computer Networks Journal on Network Modeling and Simulation, 2005.

[12] L. Kleinrock. Queueing Systems, Volume I : Theory, John Wiley, 1975.

[13] L. Kleinrock. Queueing Systems, Volume II : Computer Applications, John Wiley, 1976.

[14] R.M. Loynes. The Stability of a Queue with Non-Independent InterArrival and Service Times Proc. Cambridge Philosophical Soc. 58, 497520 .

[15] P. Nain, D. Towsley, B. Liu and Z. Liu. Properties of Random Direction Models Proc. of INFOCOM 2005, Miami, FL, March 2005.

[16] G. Sharma and R. Mazumdar. On Achievable Delay/Capacity Tradeoffs in Mobile Ad Hoc Networks Proc. of WiOpt 2004, Cambridge, UK, March 2004. 